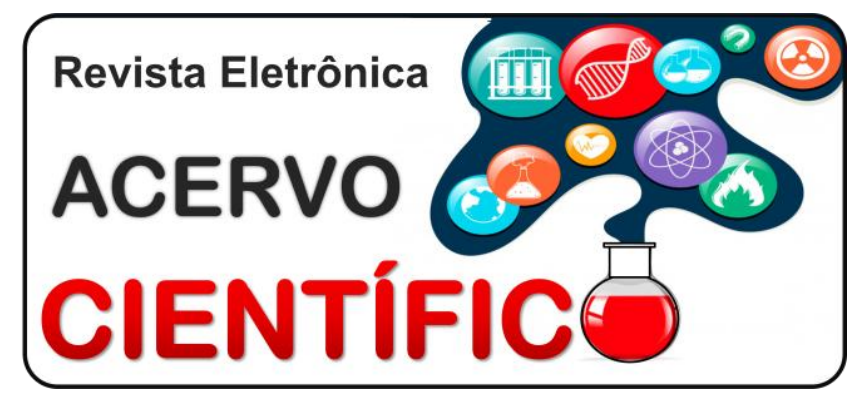

\section{ARTIGO DE REVISÃO}

Recebido em: 6/2019

Aceito em: 7/2019

Publicado em: 8/2019

\title{
A importância da anamnese na formação do acadêmico de medicina
}

The importance of anamnese in the training of the academic of medicine

La importancia de la anamnesis en la formación del académico de medicina

Luciana Regina Dias ${ }^{1}$, Osmair Alves da Silva ${ }^{1 *}$, Siandra Cordeiro Alves de Alarcão Soares ${ }^{1}$, Emílio Ernesto Garbim Junior ${ }^{1}$, Leila Rodrigues Danziger ${ }^{1}$.

Resumo: O objetivo desta pesquisa é mostrar a importância da anamnese no estudo técnico da semiologia, propondo ações que contribuam com a formação acadêmica. Para a elaboração do artigo foram consultados os principais livros de referência no assunto, mais adotados nos cursos de graduação e nos programas de residência, todos em sua edição mais recente, bem como a revisão sistemática na plataforma Scielo, dos quais foram pesquisados 177 artigos e 13 filtrados com o tema "semiologia médica", "clínica médica" e "saúde da família". A produção dos Roteiros de Semiologia e de um Anagrama esquemáticos, dividido em quatro partes; o roteiro se aplica a conceitos fundamentais com base na queixa principal e HDA. Os roteiros mostram-se como um instrumento didático de grande utilidade, facilitando o processo ensinoaprendizagem dos alunos de graduação em medicina.

Palavras-chave: Semiologia médica, Clínica médica e Saúde da família.

Abstract: The objective of this work is to show the importance of the anamnesis in a technical study of the semiology, proposing actions that contribute to the academic formation. For the elaboration of this article in which the main reference books about this subject were consulted, most adopted the undergraduate programs and the rescue programs, all in their most recent edition, articles in Scielo, 117 articles and 13 of them filtered with the theme "medical semiology" , "Medical clinic" and "family health". The production of the schematic semiology and anagram of Rotarians, divided in four parts; the roadmap applies to key concepts based on the main complaint and HDA. Rotary is presented as a useful teaching tool, facilitating the teaching-learning process of undergraduate students in medicine.

Key words: Medical semiology, Medical clinic and Family health.

Resumén: El objetivo del estudio es demostrar la importancia de la entrevista para el estudio de semiología, proponiendo acciones que contribuyan con la formación académica. Para la elaboración del artículo fueron consultados los principales libros de referencia en el asunto, cursos de graduación y programas de residencia, todos en edición más reciente, bien como una revisión sistemática de la plataforma Scielo fueron estudiados 177 artículos y 13 filtrados con el tema "semiología médica", "clínica médica" e "salud de la familia". La producción de los Procedimientos de Semiología y de un Anagrama esquemáticos, dividido en cuatro partes; el procedimiento es aplicado a conceptos fundamentales con base en la queja principal y HDA. Los procedimientos se muestran como un instrumento didáctico de gran utilidad, facilitando el proceso de aprendizaje de los alumnos de graduación en medicina.

Palabras-clave: Semiología médica, Clínica médica e Salud de la familia.

${ }^{1}$ Faculdade Morgana Potrich (FAMP), Mineiros-GO. *E-mail: osmairalves1@hotmail.com 


\section{INTRODUÇÃO}

O palavra Anamnese (do grego ana, trazer de novo e mnesis, memória) tem um significado importante nas tarefas médicas exercendo influências de forma humanizada e, colaborando com a construção de um diagnóstico pautado na queixa do paciente, desvendando a sua patologia ou doença, sempre baseada na relação médico-paciente com respeito e confiabilidade (PORTO CC, 2011).

As ciências básicas sofreram grandes transformações, principalmente na anatomia, fisiologia, patologia, farmacologia e microbiologia. Isso vem promovendo, ao final do século XIX, novas oportunidades médicas e laboratoriais, com as novas tecnologias de exames, deixando de lado a essência da anamnese. Tais acontecimentos ficam inconclusivos por falta de dados que levariam ao raciocínio clínico, e a anamnese vem para auxiliar e fechar esta lacuna, fornecendo dados que diminuam as solicitações de exames desnecessários.

A anamnese faz com que a clínica seja soberana e contribua para uma boa relação médico-paciente, levando o acadêmico de medicina a recuperar algumas qualidades que há pouco tempo estavam adormecidas, tais como: o uso da razão lógica, conhecimento de suas limitações, respeito às informações com interesse, procurar manter uma abordagem clínica sempre respeitosa, competente, segura e ética de frente a queixa principal e dados relatados pelo paciente (PORTO CC, 2017).

Entender o que significa prática médica no contexto hospitalar, acadêmico e extra-hospitalar é fundamental para o bom exercício da medicina, uma vez que a prática médica está diretamente relacionada com o que acontece no ambiente hospitalar, durante as consultas e fora deste ambiente, em que o trabalho médico está na atenção primária à saúde. Neste contexto, esta pesquisa vem para ser capaz de demostrar que os erros médicos, muitas das vezes, provocam complicações e angústias aos pacientes, que motivados por uma relação médico-paciente indesejável e defeituosa, procuram os Conselhos Regionais de Medicina (CRM), por motivos que poderiam ser resolvidos com uma boa relação (GOMES AP, 2011)

Espera-se que os futuros profissionais médicos possam demonstrar maior interesse pelo atendimento com qualidade, construindo uma boa relação médico-paciente, minimizando as decepções dos pacientes e sendo capazes de fechar um diagnóstico mais seguro, superando as tecnologias (PORTO CC, 2014).

\section{MÉTODOS}

A pesquisa consiste em uma revisão literária de livros, revistas, sites de referência e de artigos relacionados com o tema, usando a plataformas Scielo, com os seguintes descritores: semiologia médica, clínica médica e saúde da família.

Na pesquisa da plataforma foram encontrados 177 artigos e quando usado o critério dos últimos 10 anos, foram delimitados 36 artigos, destes foram usados o critério de exclusão de estudos de revisão de literatura, finalizando a busca em 13 artigos, que foram usados para embasar a pesquisa, juntamente com os outros materiais.

\section{RESULTADOS}

O Conselho Federal de Medicina (CFM) aponta três principais causas da insatisfação com a relação médico-paciente nesta era em que o individualismo predomina: influência negativa das indústrias farmacêuticas e de equipamentos, o predomínio da técnica e a soberania da vontade pessoal, que prejudica a solidariedade. Segundo a literatura pesquisada, a necessidade de aperfeiçoamento e a construção de um novo tempo se fazem necessário, para que mude o atual cenário acadêmico em meio ao fortalecimento dos laços entre o médico e o paciente (GOMES AP, 2011).

A produção e confecção de ferramentas didáticas pedagógicas, para o acadêmico do curso de medicina, desde o primeiro ano até o último, concretiza a valorização do que é aprendido desde os seus primeiros passos de aprendizagem para a sua formação, sendo um outro ponto levantado pelos autores (GOMES AP, 2011). 
Roteiros de estudos e guias bem elaborados disponibilizados através de plataformas digitais e redes sociais a nível acadêmico servem como um instrumento didático de grande utilidade, facilitando de forma gradual e contínua para o processo ensino-aprendizagem dos alunos de graduação em medicina, sendo outra forma apresentada para a qualificação do acadêmico de medicina (AGUIAR AC, 2015).

De maneira clara, significativa, objetiva e direta, porém completa, demonstrando que a semiologia é um tema de fundamental importância para o estudo, e que é essencial o entendimento dos sinais e sintomas para que o aprendizado seja significativo nas abordagens com o paciente da saúde familiar, na prática clínica, quer seja nos ambulatórios e nas visitas domiciliares, comprometendo-se a decifrar a queixa do paciente e construindo um raciocínio clínico lógico que possibilite chegar a uma hipótese diagnóstica mais pautada e que contribua para a formação do profissional médica, qualificando suas habilidades e competências (PORTO CC, 2016).

Os autores colocam o acadêmico de medicina capaz de ver a saúde como algo inerente a sua formação profissional, portanto consiga entender como acontece o processo saúde-doença e, que isso venha a fazer a diferença nos atendimentos de seus pacientes durante toda a vida. Dessa forma, o profissional será mais comprometido com a saúde, com a vida e com o outro (SOARES, MOM, et al, 2014).

\section{DISCUSSÃO}

Segundo a literatura, vários autores apontam a necessidade de criar ferramentas, roteiros, manuais e instrumentos didático-pedagógicos que contribuam para a formação do profissional de medicina. Nesse sentido, desenvolvemos três roteiros: clinica médica, pediatria, ginecologia e obstetrícia com base em um fluxograma, dividido em três partes. A primeira parte contém a Introdução da Anamnese, com um apanhado geral do roteiro a ser aplicado e seus conceitos fundamentais com base na queixa principal e HDA. A segunda parte traz os principais pontos do interrogatório dos aparelhos, composta pela anamnese com foco nos sinais e sintomas durante a entrevista (BICKLEY LS, 2015)

No tópico referente à anamnese é descrito como colher e descrever adequadamente o histórico dos sinais e sintomas os quais o paciente apresenta ao longo de sua vida, principalmente durante a consulta. $\mathrm{Na}$ relevância do roteiro são apresentados os principais conceitos semiológicos para a avaliação das funções físicas elementares do paciente e suas alterações atuais. A terceira parte dedica-se ao exame físico e a ectoscopia apresentada pelas particularidades. Além destes roteiros, uma Liga Acadêmica de Semiologia Médica (LASMED) foi criada para colocar em uso os roteiros disponibilizados por meio ao acesso às plataformas digitais, acompanhar a evolução dos acadêmicos após o uso da ferramenta de apoio para confecção de uma boa anamnese (ALVES MV, 2014). Com isso, auxiliar a construção do próprio roteiro e os Bancos de Questões levando-os para a vida. Assim, essa equipe ministrou dois minicursos para os alunos membros das LASMED e acadêmicos do curso, explicando-Ihes o funcionamento da criação (guias, roteiros em todas as cadeiras) e como eles poderão utilizar estes subsídios em prol da sua formação (PORTO CC, 2017).

\section{CONSIDERAÇÕES FINAIS}

O roteiro tem por finalidade ser um instrumento didático de grande valia para a formação acadêmica. Desse modo, facilitar gradualmente o processo ensino-aprendizagem dos alunos de graduação em medicina. De maneira clara, objetiva, direta e completa, demonstrar que a anamnese bem-feita é de fundamental importância no estudo e entendimento dos sinais e sintomas do paciente para o aprendizado significativo das abordagens com o paciente da saúde familiar na prática clínica de ambulatório e nas visitas domiciliares. Com isso comprometendo-se na construção do raciocínio clínico na formação do profissional médico.

\section{AGRADECIMENTOS E FINANCIAMENTO}

A toda parte docente que contribuiu durante toda a nossa formação. E aos nossos orientadores que não mediram esforços para elaboração e concretização desse trabalho. 


\section{REFERÊNCIAS}

1. AGUIAR AC, RIBEIRO ECO. Conceito e avaliação de habilidades e competência na educação médica. Rev. bras. educ. med 2015; 34(3): 371-378.

2. ALVES MV. A Faculdade de Medicina da Universidade de Lisboa - Um olhar sobre a sua história. Lisboa, Gradiva, 2011.

3. ALVES MV. História da Medicina em Portugal - Origens, ligações e contextos. Porto, Porto Editora, 2014.

4. AYRES JRCM. O cuidado, os modos de ser (do) humano e as práticas de saúde, Saúde e Sociedade v.13, n.3, p.1629, set-dez 2004.

5. BICKLEY LS. Bates - Propedêutica Médica - Lynn S. Bickley. 11aㅡ Edição. 2015. Editora Guanabara Koogan.

6. BRASIL, Cadernos de Atenção Básica: Diretrizes do NASF. Ministério da Saúde. Secretaria de Atenção à Saúde. Departamento de Atenção Básica. Saúde na escola / Ministério da Saúde, Secretaria de Atenção à Saúde, Departamento de Atenção Básica. - Brasília: Ministério da Saúde, 2009. (160p. versão preliminar).

7. BRASIL. Ministério da Educação. Conselho Nacional de Educação, Câmara de Educação Superior. Resolução CNE/CES n4 de 7 de novembro de 2001. Institui diretrizes curriculares nacionais do curso de graduação em Medicina. Diário Oficial da União. Brasília, 9 nov. 2001; Seção 1, p.38.

8. BRASIL. Ministério da Saúde. Secretaria de Atenção à Saúde. Departamento de Atenção Básica. SIAB: manual do sistema de Informação de Atenção Básica /Ministério da Saúde, Secretaria de Atenção à Saúde, Departamento de Atenção Básica. - 1. R., 4. ${ }^{a}$ reimpr. - Brasília: Ministério da Saúde, 2003.

9. BUZZI A. La entrevista terapéutica. Rev Asoc Méd Argent. 2009; 122(3):14-21.

10. CAMPOS GWS. Equipes de referência e apoio especializado matricial: um ensaio sobre a reorganização do trabalho em saúde, Ciência \& Saúde Coletiva, 4(2):393-403, 1999.

11. CAPRA F. O ponto da mutação: a ciência, a sociedade e a cultura emergente. 2006. São Paulo: Cultrix.

12. DICHI JB, DICHI I. Agonia da história clínica e suas consequências para o ensino médico. Rev Bras Educ Méd. 2006; 30(2):93-7.

13. FERREIRA AB, et al. Propedêutica médica da criança ao idoso. 2. ed. São Paulo: Editora Atheneu, 2015.

14. GOMES AP, REGO S. Transformação da educação médica: é possível formar um novo médico a partir de mudanças no método de ensino-aprendizagem? Rev. bras. educ. med. 2011; 35(4): 557-566.

15. HIPÓCRATES. Aforismos. São Paulo: Editora Unifesp, 2010

16. HIPPOCRATIC Writings. Enclyclopaedia Britannica INC. Chicago: 1952.

17. LOPÉZ M. Anamnese. In: Lopéz M, Laurentys M, organizadores. Semiologia médica: as bases do diagnóstico clínico. 5. ed. Rio de Janeiro: Revinter; 2004. p. 23-38.

18. LUCCA SR, KITAMURA S. O ensino da Medicina do Trabalho e a importância das visitas aos locais de trabalho. Rev Bras Med Trab. 2012; 10(2):41-8.

19. PEREIRA OP, ALMEIDA TMC. A formação médica segundo uma pedagogia de resistência. Interface- Comunic., Saúde, Educ. 2005; 19(16):69-79.

20. PORTO CC. Clínica Médica na Prática Diária. Rio de Janeiro: Guanabara-Koogan. 2016. 1ª Edição, 1482 p.

21. PORTO CC. Doenças do Coração - Prevenção e Tratamento. 2ª ed. Rio de Janeiro: Guanabara-Koogan, 2005. $1118 \mathrm{p}$.

22. PORTO CC. Exame Clínico - Bases para a Prática Médica. 8ª ed. Rio de Janeiro: Guanabara-Koogan, 2017. 544 páginas.

23. PORTO CC. Semiologia Médica - Mario López, José Laurentys Medeiros - 5a Edição. 2009. Editora Atheneu.

24. PORTO CC. Vademecum de Clínica Médica. Rio de Janeiro: Guanabara-Koogan, 3ª Edição. 2010. 1070 p

25. PORTO CC, PORTO AL. Semiologia Médica. 7ª̣ ed. Rio de Janeiro: Guanabara-Koogan, 2014. 1320 p

26. RIBEIRO MMF, AMARAL CFS. Medicina centrada no paciente e o ensino médico: importância do cuidado com a pessoa e o poder médico. Rev Bras Educ Med.2008; 32(1):90-7.

27. RONAN CA. História ilustrada da ciência da Universidade de Cambridge (4 V) vol I: Das origens à Grécia; vol II: Oriente, Roma e idade média. RJ, Zahar, 1987.

28. ROSENBAUM P. Novissima Medicina. São Paulo: Ed. Organon, 2008.

29. SIQUEIRA-BATISTA R. Aprendizagem baseada em problemas: uma estratégia das sociedades de controle 2006. [trabalho de conclusão de curso]. Curso de especialização em Ativação de Processos de Mudança na Formação Superior de Profissionais de Saúde, Fundação Oswaldo Cruz; sobre as sociedades de controle. In: Deleuze G. Conversações: 1972-1990. Rio de Janeiro: Editora. Lisboa: Edições.

30. SOARES, MOM et al. Reflexões contemporâneas sobre anamnese na visão do estudante de medicina. Rev. bras. educ. med. [online]. 2014, vol.38, n.3, pp.314-322. ISSN 0100-5502. 